\title{
Anti-windup for model-reference adaptive control schemes with rate-limits ${ }^{\text {th }}$
}

\author{
Matthew C. Turner ${ }^{\mathrm{a}, *}$, Jorge Sofrony ${ }^{\mathrm{c}}$, Emmanuel Prempain $^{\mathrm{b}}$ \\ ${ }^{a}$ School of Electronics and Computer Science, University of Southampton, Southampton, SO17 1BJ. \\ ${ }^{b}$ Department of Engineering, University of Leicester, Leicester, LE1 7RH, UK. \\ ${ }^{c}$ Department of Mechatronics, Nacional University of Colombia, Bogota, Colombia
}

\begin{abstract}
This paper proposes an anti-windup like scheme for an LTI plant with rate-limits. The plant is controlled using a model-reference adaptive controller, making the anti-windup design problem highly nonlinear. It is assumed that the rate-limit is modelled as a first order feedback loop for which the state is unavailable, but that the bandwidth of this loop is known. The anti-windup scheme uses a "hedging" term and a "positive $\mu$ " term. The structure of the problem makes the rate-limit case considerably more difficult than the magnitude limit case. Nevertheless it is proved that convergence of the system state to the ideal model can be accomplished under conditions similar to those found in anti-windup compensation for purely linear systems.
\end{abstract}

Keywords: adaptive control, anti-windup, saturation

\section{INTRODUCTION}

Control constraints, an ever-present nonlinearity, are often a thorn in the side of control engineers, sometimes causing well-behaved systems to exhibit unexpectedly poor behaviour. They have been tackled using various approaches in the control systems literature, with the earliest approach probably being the anti-windup approach used successfully in industry. The idea behind anti-windup is to introduce an extra element into the control system which monitors the control signal and, if it experiences saturation, modifies the control system so that it behaves better during and after these periods of saturation. An enormous amount of research activity was devoted to anti-windup in the late 1990s and 2000s. The main thrust of this research was to develop more systematic anti-windup approaches which could guarantee stability of the closed-loop system

\footnotetext{
${ }^{2}$ Research supported by the UK EPSRC Grant number EP/N00924X/1

* Corresponding author

Email addresses: M. C. Turner@soton. ac.uk (Matthew C. Turner), 
during saturation and also to ensure that performance did not dramatically deteriorate - see $[4,18,25,3,19]$.

A common feature in much of the anti-windup literature is that most of the available schemes are model-based, with their design depending on the system parameters. Most of the modern anti-windup methods frame the anti-windup design problem as linear matrix inequality feasibility/optimisation problems which necessarily entail a reasonable knowledge of the plant parameters. This is in stark contrast to the more traditional anti-windup approaches which were $a d$ hoc and rarely dependent on plant parameters.

In model reference adaptive control (MRAC), it is typically assumed that only the structure of the plant is known, with many of its parameters unknown. For this reason the anti-windup schemes described above are not appropriate, without significant alteration. In addition, the problem with traditional anti-windup schemes (i.e. the ad hoc nature and lack of rigour) are even more troublesome for adaptive controllers due to their greater complexity, and also because saturation may corrupt both the control law itself and the adaptive up-dating mechanism. Therefore techniques for handling saturation in adaptive controllers, with some exceptions such as [9], which is applicable to indirect adaptive control schemes, have followed a somewhat different path. Notable techniques include $[10,8,1,23,13,20]$. These schemes do not act like antiwindup compensators in the sense that an anti-windup compensator is not activated solely during periods of saturation, with control handled by the baseline controller at other times.

Unlike most of the approaches for handling saturation in adaptive controllers mentioned above, the so-called positive- $\mu$ scheme described in [14] (see also [24]), functions somewhat differently and, when expressed appropriately, one can observe an anti-windup structure in the algorithm [21]. This anti-windup scheme features a classical anti-windup element (the $\mu$ parameter) which feeds back the difference between the control signal and its saturated version to the controller output. This same signal also modifies the on-line model states and introduces an additional adaptive parameter which is adjusted during periods of saturation. The functionality of the scheme is relatively simple, somewhat intuitive to tune, and fits snugly within the anti-windup framework.

One limitation with the positive- $\mu$ scheme is that it is applicable to systems with control magnitude limits; it is not "out of the box" applicable to systems with rate-limited actuators. In certain control problems such as flight control law design, actuator ratelimits are of more concern than magnitude limits. It therefore makes sense to try to extend some of the ideas of the positive- $\mu$ approach to systems with rate-limited actuators. There are a number of technical issues with such an extension, however. One of these issues centres around the measurement of the states: MRAC assumes that all states are available for measurement, but it is typically unlikely that the actuator states will be available for measurement, or even desirable to use in the adaptive controller. Furthermore, it transpires, that even with the assumption of rate-limit state measurement, the resulting structure of the system then becomes too limiting, with unrealistic structural requirements placed on the plant and model.

With the above in mind, the aim of this paper therefore is to devise an anti-windup 
scheme for MRAC schemes with rate-limited actuators. The idea is to maintain the spirit of the original positive- $\mu$ scheme but to modify it so that it can cope with ratelimits instead of magnitude limits. The cost of making this extension is that some of the anti-windup properties proved in [21] are no longer retained in general, although the hope is that the intuition in the scheme remains. For simplicity, this paper considers SISO systems, although an extension to MIMO systems could be performed using similar ideas.

\subsection{Notation}

Notation is standard throughout. A positive (negative) definite matrix $M$ is denoted $M>0(<0)$. The scalar saturation function $\operatorname{sat}_{\bar{u}}():. \mathbb{R} \mapsto[-\bar{u}, \bar{u}]$ is defined as

$$
\operatorname{sat}_{\bar{u}}(u)=\operatorname{sign}(u) \min \{|u|, \bar{u}\} \quad \bar{u}>0
$$

The scalar deadzone function $\mathrm{Dz}_{\bar{u}}():. \mathbb{R} \mapsto \mathbb{R}$ is defined as

$$
\operatorname{Dz}_{\bar{u}}(u)=\operatorname{sign}(u) \max \{0,|u|-\bar{u}\} \quad \bar{u}>0
$$

The saturation and deadzone functions satisfy the identity

$$
\operatorname{sat}_{\bar{u}}(u)+\mathrm{Dz}_{\bar{u}}(u)=u
$$

Note that both functions are globally Lipschitz with a Lipschitz constant of unity, meaning that

$$
\left\|\phi\left(x_{1}+x_{2}\right)-\phi\left(x_{1}\right)\right\| \leq\left\|x_{2}\right\| \quad \forall x_{1}, x_{2} \in \mathbb{R}
$$

where $\phi($.$) is either the saturation or the deadzone function and \|\cdot\|$ denotes the Euclidean norm.

A signal $x(t)$ is said to belong the Lebesgue space $\mathcal{L}_{2}$ if its $\mathcal{L}_{2}$ norm is finite, i.e.

$$
\|x\|_{2}:=\left(\int_{0}^{\infty}\|x(t)\|^{2} d t\right)^{\frac{1}{2}}<\infty
$$

Similarly a signal is said to belong to the Lebesgue space $\mathcal{L}_{\infty}$ if its $\mathcal{L}_{\infty}$ norm is finite, i.e.

$$
\|x\|_{\infty}:=\sup _{t \geq 0} \max _{i}\left|x_{i}(t)\right|<\infty
$$

\section{PRELIMINARY RESULTS}

Several results will be used in the proof of the main results. Most of these are standard results in nonlinear control [11], but there are two particular results which are introduced below. 
Lemma 1. Consider the dynamics

$$
\dot{x}(t)=A x(t)+B w(t) \quad x(t) \in \mathbb{R}, \quad w(t) \in \mathbb{R}
$$

If $A$ is Hurwitz and $w(t)$ is bounded, then the state $x(t)$ is bounded.

Proof: In essence, this result is a well-known fact. It is proved in the first part of Theorem 1 in [14]; see also Section 6, [12].

Lemma 2. Consider the dynamics

$$
\dot{x}=-\lambda x+\operatorname{sat}_{\bar{u}}(w)
$$

where $x(t), w(t) \in \mathbb{R}$ and $\lambda>0$ is a positive scalar. Then $x(t)$ is bounded.

Proof: This is a corollary of Lemma 1: $\operatorname{sat}_{\bar{u}}(w)$ is bounded, regardless of $w(t)$.

Another standard fact will also be useful.

Fact 1. Let $\phi():. \mathbb{R} \mapsto \mathbb{R}$ be a slope-restricted nonlinearity with slope $\partial \phi \in[0,1]$; this means that it satisfies the relation

$$
0 \leq \frac{\phi\left(u_{1}\right)-\phi\left(u_{2}\right)}{u_{1}-u_{2}} \leq 1 \quad \forall u_{1}, u_{2} \neq u_{1}
$$

Then the following inequality holds:

$$
\left(\phi\left(u_{1}\right)-\phi\left(u_{2}\right)\right)\left[u_{1}-u_{2}-\left(\phi\left(u_{1}\right)-\phi\left(u_{2}\right)\right)\right] \geq 0
$$

The following lemma from [6] (Lemma 3.2.5) will be used later in the paper. It is a special case of the more general Barbalat's Lemma.

Lemma 3. Consider the function $w(t)$. If $w(t), \dot{w}(t) \in \mathcal{L}_{\infty}$ and $w(t) \in \mathcal{L}_{2}$ then $\lim _{t \rightarrow \infty} w(t)=0$.

The first two conditions, i.e. $w(t), \dot{w}(t) \in \mathcal{L}_{\infty}$, guarantee uniform continuity of $w(t)$, with the $\mathcal{L}_{2}$ property guaranteeing convergence. The following simple lemma, proved in the appendix, is useful for the technical results reported in the paper

Lemma 4. Assume $\pi():.[0, \infty) \mapsto[0, \delta]$, then the following inequality holds

$$
\left\|\mathrm{Dz}_{\bar{u}}[\pi(t) u]\right\| \leq\left\|\mathrm{Dz}_{\bar{u}}[\delta u]\right\| \quad \forall u \in \mathbb{R}
$$


The final preliminary result which is needed is an adaption of the Comparison Lemma ([12] Lemma 2.5); the appendix contains a proof for completeness.

Lemma 5. Consider the quadratic function $V(x(t))=x(t)^{\prime} P x(t)$, where $x(t) \in \mathbb{R}^{n}$, and the following inequality

$$
\dot{V}(x(t)) \leq-\alpha_{1}\|x(t)\|^{2}+\alpha_{2}\|x(t)\| \sum_{i=1}^{m}\left\|u_{i}(t)\right\|
$$

where $\alpha_{1}$ and $\alpha_{2}$ are positive constants. Then if $u_{i}(t) \in \mathcal{L}_{2}$ for all $i \in 1, \ldots, m$, $\lim _{t \rightarrow \infty} x(t)=0$.

\section{Problem Formulation}

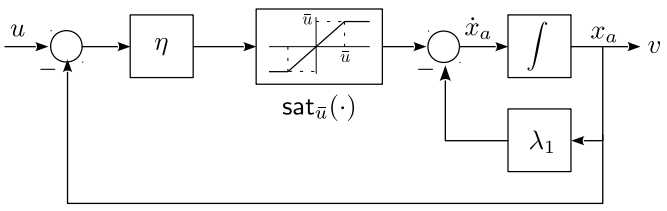

Figure 1: Model of actuator with rate-limit: $\eta$ determines the actuator bandwidth and $\lambda_{1}$ represents the integrator leakage.

The plant dynamics under consideration are given below.

$$
\dot{x}=A x+B v
$$

where $A \in \mathbb{R}^{n \times n}$ and $B \in \mathbb{R}^{n}$; it is assumed that the states are available for feedback. The input to the plant is the signal $v(t) \in \mathbb{R}$ which is the output of the (rate-limited) actuator dynamics, illustrated in Figure 1, and given below

$$
\begin{aligned}
\dot{x}_{a} & =-\lambda_{1} x_{a}+\operatorname{sat}_{\bar{u}}\left[\eta\left(u-x_{a}\right)\right] \\
v & =x_{a}
\end{aligned}
$$

Here, $u(t) \in \mathbb{R}$ is the "real" control input, $\eta>0$ is the actuator bandwidth and the saturation function models the rate-limits. The actuator is assumed to have a "leaky integrator" with $\lambda_{1} \ll 1$. In the limit as $\lambda_{1} \rightarrow 0$, one recovers the typical first order rate-limit model used frequently in the anti-windup literature [7, 2, 17]. This is done mainly to facilitate the proof of convergence of the closed loop system; it is well known that the presence of integrators in the plant can complicate stability analysis of saturated systems and a body of work has been dedicated to this topic e.g. [22, 15]. The system can be represented compactly as

$$
\begin{aligned}
\dot{x} & =A x+B x_{a} \\
\dot{x}_{a} & =-\lambda_{1} x_{a}+\operatorname{sat}_{\bar{u}}\left[\eta\left(u-x_{a}\right)\right]
\end{aligned}
$$

It is important to mention the fact that the actuator state $x_{a}$ (i.e. the plant input $v(t)$ ) are not directly measured, hence they must be estimated. 
Assumption 1. $A \in \mathbb{R}^{n \times n}$ is unknown but Hurwitz; $B \in \mathbb{R}^{n}$ is known; $\eta, \lambda_{1} \in \mathbb{R}_{+}$ are known.

If $A$ is not Hurwitz, it is well-known that global asymptotic stability cannot be established for a plant with input constraints. In this case, the region of attraction [7] becomes of interest. However, estimation of the region of attraction requires precise knowledge about $A$ and it is therefore difficult to propose a rigorous anti-windup technique for non-Hurwitz $A$ in the MRAC framework.

Remark 1: The results of the paper are derived assuming that the plant states are available, but the actuator state is not. Typically, actuator states are not available for feedback, or at least they are not available to the main control system, whereas data modelling, approximately, an actuator's dynamic characteristics are often available. This assumption means that there are non-trivial technical differences between the results derived here and those obtained in [21], as will be seen by a careful perusal of the proofs which follow.

The reference model (see Figure 2) used for the adaptive controller is

$$
\dot{x}_{m}=A_{m} x_{m}+B_{m} r+B\left(\hat{x}_{a}-u\right)-B \mu \mathrm{Dz}_{\bar{u}}\left[\eta\left(u-\hat{x}_{a}\right)\right]-B \hat{K}_{u} \mathrm{Dz}_{\bar{u}}\left[\eta\left(u-\hat{x}_{a}\right)\right]
$$

where $x_{m} \in \mathbb{R}^{n}$ is the model state, $r \in \mathbb{R}$ the reference to be tracked, $\hat{x}_{a} \in \mathbb{R}$ the estimate of the actuator state (defined below) and $\hat{K}_{u}(t) \in \mathbb{R}$ an adaptive gain. $A_{m}$ and $B_{m}$ are known matrices representing the ideal system (introduced below) and $\mu \in \mathbb{R}_{+}$ is a positive scalar chosen by the designer. The idea with this on-line model is that if the rate-limit is not active, that is if $\operatorname{Dz}_{\bar{u}}\left[\eta\left(u-\hat{x}_{a}\right)\right] \equiv 0$ and if the actuator state perfectly matches the control input (during constant control activity for example), the dynamics of the model reduce to

$$
\dot{x}_{m}=A_{m} x_{m}+B_{m} r
$$

which is the standard reference model used in MRAC. The extra terms are present to accommodate the rate-limited actuator dynamics. The third term on the r.h.s. of

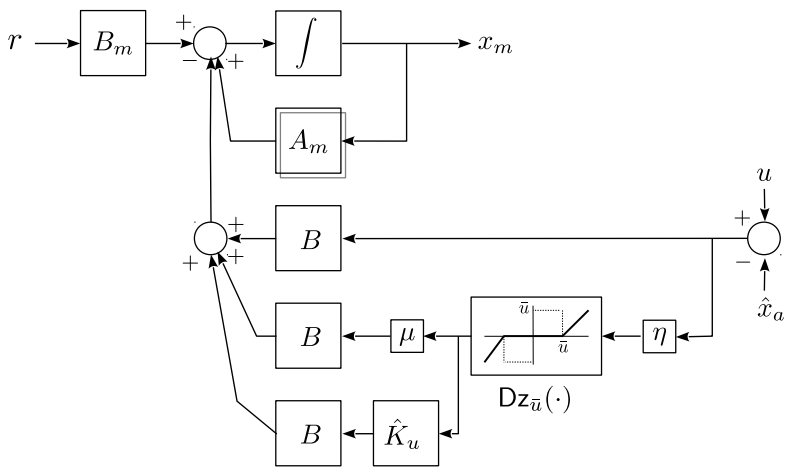

Figure 2: Modified reference model used in adaptive control law. 
equation (12) is a "hedging term" ([8, 5]) and is useful to prove convergence, but is also problematic because only at steady state will $\hat{x}_{a}$ converge to $u$. The effect of the hedging term will be discussed later.

A crucial contributor to the reference model (12) is the actuator state estimator (see Figure 3), which has the following dynamics,

$$
\dot{\hat{x}}_{a}=-\lambda_{1} \hat{x}_{a}+\operatorname{sat}_{\bar{u}}\left[\eta\left(u-\hat{x}_{a}\right)\right]+B^{\prime} P e
$$

where $e=x-x_{m}$ and $P=P^{T}$ is the solution to the Lyapunov equation:

$$
A_{m}^{\prime} P+P A_{m}=-Q<0, \quad Q>0
$$

The control signal is given by

$$
u=\hat{K}_{x}^{\prime} x+\hat{K}_{r}^{\prime} r-\mu \mathrm{Dz}_{\bar{u}}\left[\eta\left(u-\hat{x}_{a}\right)\right]
$$

where $\hat{K}_{x}(t) \in \mathbb{R}^{n}$ and $\hat{K}_{r}(t) \in \mathbb{R}$. This control signal has a similar form to that in [21], except that the additional portion - the anti-windup term involving $\mu$ - is activated when the rate-limit saturates (instead of when the magnitude limit saturates). The next assumption is standard in MRAC

Assumption 2. There exist matrices $K_{x}^{*}$ and $K_{r}^{*}$ such that

$$
\begin{aligned}
& A_{m}=A+B K_{x}^{*} \\
& B_{m}=B K_{r}^{*}
\end{aligned}
$$

Assummption 2 restricts freedom in the choice of reference model and is a well-known limitation of MRAC. Similar to anti-windup for linear systems, the anti-windup modification proposed in this paper is carried out on the basis of a "good" existing (MRAC) controller design. Therefore, in the sections which follow, the modified adaptive controller which is given necessarily requires that Assumptions 1 and 2 are satisfied.

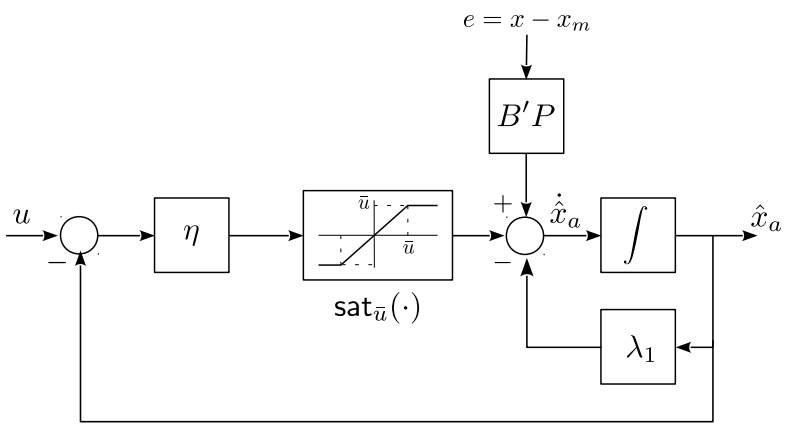

Figure 3: Rate-limit state-estimator for use with modified reference model (state of actuator is assumed not to be available directly). 


\section{MAIN RESULTS}

This section presents an adaptive update law which is used in conjunction with controller (16) to prove that the plant states, $x$, converge to those of the reference model $x_{m}$. Comments will also be made about the possibility of convergence to an ideal model, without additional terms. Before stating the main result, it is convenient to note that the control law (16) can be re-written as

$$
u=K_{x}^{*} x+K_{r}^{*} r+\Delta K_{x}^{\prime} x+\Delta K_{r}^{\prime} r-\mu \mathrm{Dz}_{\bar{u}}\left[\eta\left(u-\hat{x}_{a}\right)\right]
$$

where

$$
\begin{aligned}
\Delta K_{x}^{\prime} & :=\hat{K}_{x}^{\prime}-K_{x}^{*} \\
\Delta K_{r}^{\prime} & :=\hat{K}_{r}^{\prime}-K_{r}^{*}
\end{aligned}
$$

and $K_{x}^{*}$ and $K_{r}^{*}$ are the matrices mentioned in Assumption 2. Similar to [14, 21], the adaptive updates are modified to incorporate an additional adaptive term which is dependent on whether the synthetic rate-limit (14) is saturated or not, viz

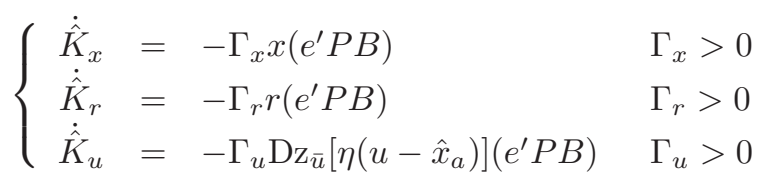

The first two updates are standard ([6]); the third is inspired by [14], and is only active if rate saturation occurs. The main result of the paper is the following proposition.

Proposition 1. Consider the interconnection of the plant (10), the rate limits (11), the controller (16), the update law (22), and the dynamics (12) and let Assumptions 1 and 2 be satisfied. Assume further that $\mu \in \mathbb{R}_{+}$and that $r \in \mathcal{L}_{\infty}$. Then the signals $e=$ $x-x_{m}, e_{a}=x_{a}-\hat{x}_{a}, \hat{K}_{x}, \hat{K}_{r}$ and $\hat{K}_{u}$ are all bounded. Furthermore $\lim _{t \rightarrow \infty} e(t)=0$ and $\lim _{t \rightarrow \infty} e_{a}(t)=0$.

Proof: Please see appendix for full proof. The main idea of the proof is to use a Lyapunov function to prove boundedness of $e(t), e_{a}(t)$ and the adaptive gains and then to use Lemma 3 to prove that $e(t)$ and $e_{a}(t)$ converge - in a similar way to standard MRAC proofs. The technical complications in the proof arise to the presence of the anti-windup compensator and the saturation nonlinearity: Facts 1 and Lemmas 1 and 2 allow these obstacles to be overcome.

Remark 2: Proposition 1 also holds when the adaptive gain $\hat{K}_{u}(t)$ is absent; that is the errors $e(t)$ and $e_{r}(t)$ converge if $\hat{K}_{u} \equiv 0$. However, the presence of $\hat{K}_{u}(t)$ provides more freedom for the anti-windup compensator to modify the system's behaviour during saturation, and it seems to have a favourable effect on the transient response of the system. This is demonstrated in simulation later in the paper. 


\subsection{Convergence to ideal behaviour}

In [21] it was shown how, under conditions similar to those found in linear anti-windup - that is, that the ideal control law was not saturated in steady state - the state of the system was actually able to converge to the ideal reference model

$$
\dot{x}_{r}=A_{m} x_{r}+B_{m} r
$$

[21] considered the magnitude limit problem and unfortunately, for the rate-limit problem, considered here, the results do not carry over in a straightforward manner. This difficulty arises, essentially, because the rate-limit dynamics prevent plant input $v$ from converging to the ideal control signal except if the ideal control signal is constant. However, convergence to ideal behaviour can be proved if we modify our expectations a little. With this in mind, we therefore propose the following ideal model which we would like our constrained adaptive system to converge to

$$
\left\{\begin{array}{l}
\dot{x}_{r}=A_{m} x_{r}+B_{m} r+B\left(\hat{x}_{a r}-u\right) \\
\dot{\hat{x}}_{a r}=-\left(\lambda_{1}+\eta\right) \hat{x}_{a r}+\eta u
\end{array}\right.
$$

The $x_{r}$ dynamics represent the ideal behaviour of our plant state; note that in steady state if $\hat{x}_{a r} \rightarrow u$, this becomes our initial choice for ideal system (23). The $\hat{x}_{a r}$ dynamics represent the ideal dynamics of the rate-limit and is essentially the rate-limit model earlier sans the saturation nonlinearity. It is convenient to note the following fact.

Fact 2. Consider the dynamics (24). Then it follows that

$$
\left\|\hat{x}_{a r}\right\|_{\infty} \leq \frac{\eta}{1+\eta}\|u\|_{\infty} \quad \forall \eta>0
$$

and furthermore $\hat{x}_{a r}(t)=K(t) u(t)$ for some $K(t) \in[-1,1]$

Proposition 1 has already proved that $x \rightarrow x_{m}$ (under certain assumptions) and $x_{a} \rightarrow$ $\hat{x}_{a}$, so it follows that ideal behaviour, will therefore result if

$$
x_{m} \rightarrow x_{r} \quad \text { and } \quad \hat{x}_{a} \rightarrow \hat{x}_{a r}
$$

Stated another way, we would like to enforce

$$
\begin{aligned}
\lim _{t \rightarrow \infty} e_{m}(t) & =0 & e_{m}:=x_{m}-x_{r} \\
\lim _{t \rightarrow \infty} e_{r}(t) & =0 & e_{r}:=\hat{x}_{a}-\hat{x}_{a r}
\end{aligned}
$$

The next proposition gives conditions which ensure this convergence takes place. The proposition is stated in terms of certain signals belonging to the space $\mathcal{L}_{2}$. In particular, it is covenient to partition the control signal as

$$
u=\kappa(u)(\underbrace{K_{x}^{*} x+K_{r}^{*} r}_{=u^{*}(t)}+\underbrace{\Delta K_{x}^{\prime} x+\Delta K_{r}^{\prime} r}_{=\Delta u(t)})
$$


in the same way as in [21]. In this partition, $u^{*}(t)$ represents the ideal control signal (i.e. that which would be applied if the ideal gains $K_{x}^{*}$ and $K_{r}^{*}$ were applied) and $\Delta u(t)$ represents the perturbation from this ideal, due to the adaptation. These two signals play a central role in the proposition below.

Proposition 2. Consider the interconnection of the plant (10), the rate limits (11), the controller (16), the update law (22), and the dynamics (12), (24) and let Assumptions 1 and 2 be satisfied. Assume further that $\mu \in \mathbb{R}_{+}$and that $r \in \mathcal{L}_{\infty}$. Then $\lim _{t \rightarrow \infty} e_{m}(t)=0$ and $\lim _{t \rightarrow \infty} e_{r}(t)=0$ if the following conditions are satisfied

1. $\mathrm{Dz}_{\bar{u}}\left(2 \eta u^{*}(t)\right) \in \mathcal{L}_{2}$

2. $\Delta u(t) \in \mathcal{L}_{2}$

Proof: Please see appendix for full proof. The main idea is to use Lyapunov functions for the dynamics governing the errors $e_{m}(t)$ and $e_{r}(t)$ and then to apply the comparison lemma (Lemma 5) to these inequalities to obtain Conditions 1 and 2 above, which are the conditions for convergence. Lemma 4 is required to treat the deadzone and Fact 2 is need to bound the rate-limit state estimation.

In a nutshell, Proposition 2 proves that the state of the system and actuator will converge to the "ideal" states defined by the model (24) if $\mathrm{Dz}\left(2 \eta u^{*}\right) \in \mathcal{L}_{2}$. Roughly speaking, for most signals this effectively means that convergence will occur if, in steady state we have the signal satisfying

$$
\left|\eta u^{*}\right|<\frac{1}{2} \bar{u}
$$

Essentially, this means that the signal entering the saturation block in the rate-limit must converge to half the limit of the saturation signal, which is stronger than requiring

$$
\left|\eta u^{*}\right|<\bar{u}
$$

However, it has not been possible to prove the former relaxed bound because of the presence of the dynamics of the rate-limit. Nevetheless, this bound is again reminiscent of what would be expected in anti-windup for linear controllers with rate-limits.

Remark 3: In a similar way to [21], it is possible to prove Proposition 2 under slightly different conditions which make it more applicable in practice. Note that Proposition 2 requires that $\Delta u(t) \in \mathcal{L}_{2}$, which effectively requires $\hat{K}_{x}$ to converge to its ideal value, which it may not always do - it is only known that it is bounded. To overcome this restriction, one may instead partition the control signal as

$$
u=\kappa(u)\left[u^{*}+\Delta u_{s s}+\Delta u-\Delta u_{s s}\right]
$$

where

$$
\Delta u_{s s}(t):=\Delta \hat{K}_{x, s s} x(t)+\Delta \hat{K}_{r, s s} r(t)
$$


and

$$
\begin{aligned}
\lim _{t \rightarrow \infty} \Delta K_{x}(t) & :=\Delta K_{x, s s} \\
\lim _{t \rightarrow \infty} \Delta K_{r}(t) & :=\Delta K_{r, s s}
\end{aligned}
$$

In other words $\Delta K_{x, s s}$ and $\Delta K_{r, s s}$ indicate that, in steady state, the adaptive gains will converge to a value, probably different from zero. In this case, the conditions on Proposition 2 can be changed to

1. $\mathrm{Dz}_{\bar{u}}\left[2 \eta\left(u^{*}(t)+\Delta u_{s s}(t)\right] \in \mathcal{L}_{2}\right.$

2. $\Delta u(t)-\Delta u_{s s}(t) \in \mathcal{L}_{2}$

This means that we simply require the ideal value of the saturation input - plus some perturbation - to fall below $1 / 2 \bar{u}$ and that the adaptive gains converge to their steady state values. The latter condition is much more typical in practice.

\section{SIMULATION RESULTS}

The results are demonstrated using an example proposed in [16], and also used in [21] to illustrate an anti-windup scheme for MRAC systems with magnitude limits. The plant state-space matrices are given by:

$$
A=\left[\begin{array}{ccc}
0 & 1 & 0 \\
-10 & -1.167 & 25 \\
0 & 0 & -0.8
\end{array}\right] \quad B=\left[\begin{array}{c}
0 \\
0 \\
2.4
\end{array}\right]
$$

A nominal MRAC controller was found by choosing $\Gamma_{x}=0.5 I_{3}$ and $\Gamma_{u}=0.5$. The state-space matrices of the reference model were chosen as

$$
A_{m}=\left[\begin{array}{ccc}
0 & 1 & 0 \\
-10 & -1.167 & 25 \\
1.933 & -0.974 & -10.833
\end{array}\right] \quad B_{m}=B
$$

It is easy to see that Assumption 2 is satisfied. With $Q=I_{3}, P>0$ was was calculated from (15). The choice of $\Gamma_{x}, \Gamma_{u}$ and $Q$ was based on some initial simulation and was straightforward; it seemed to enable swift adaptation but without too much control activity; other choices are of course possible. This adaptive controller works well in the absence of saturation, but the system is very sensitive to saturation [16].

The plant above was augmented with the first order actuator model discussed earlier. The bandwidth of the actuator, set by the parameter $\eta$, was set at $100 \mathrm{rad} / \mathrm{s}$. The leakage term $\lambda_{1}$ of the plant actuator was actually set to zero i.e. the rate-limit was chosen to be a standard first order rate-limited actuator. The anti-windup scheme was designed using Proposition 1 with $\mu=0.5$ and the anti-windup adaptive weight, $\Gamma_{u}=0.5$. Simulations revealed that larger values for $\mu$ tended to lead to more oscillatory transient responses; the system was fairly insensitive to the choice of $\Gamma_{u}$, which was chosen to be consistent with $\Gamma_{x}$ and $\Gamma_{r}$. 
Figure 4 shows the response of the unconstrained system to reference which consists of a step input of $20[\mathrm{~cm}]$ applied at 1 [sec], followed by a step down to $10[\mathrm{~cm}]$, a step down to $4[\mathrm{~cm}]$ and then a ramp to $2[\mathrm{~cm}]$. The simulation was conducted with the first order actuator dynamics present, i.e. the rate-limit was just a first order lowpass filter. One can note that the system responds generally satisfactorily, although it is noted that there is a great deal of control activity which may cause problems when ratelimiting is introduced. Indeed, when rate-limits of $100 \mathrm{~V} / \mathrm{s}$ are imposed on the system, the response degrades to that shown in Figure 5. Note the control signal displays the typical triangular wave form indicative of extensive rate-saturation.

Figure 6 shows the response of the system with rate-limits present but with the antiwindup compensator described above implemented in addition to the nominal MRAC controller. The response of the system is much improved and nominal performance has almost been recovered. Interestingly, one might argue that the response is, in some ways, superior even to the nominal response shown in Figure 4: during the initial phase of adaptation the rate-limited MRAC with anti-windup shows lower control activity and the convergence of the states to the model values is rather less oscillatory. However, the control signal does show high frequency behaviour which takes some time to decay: recall no explicit decay rate for the system has been found - this issue could be overcome in practice through the use of sigma modification.

The anti-windup method proposed here adds an adaptive gain $\hat{K}_{u}(t)$ to the control system but, as noted in Remark 2, the approach can work without this and with only the parameter $\mu$ present. However, this deprives the anti-windup compensator of a useful degree of freedom and one would expect the performance of the compensator to be worse, in some sense. Figure 7 confirms this, with both the plant and control response begin somewhat more oscillatory without the presence of $\hat{K}_{u}(t)$.
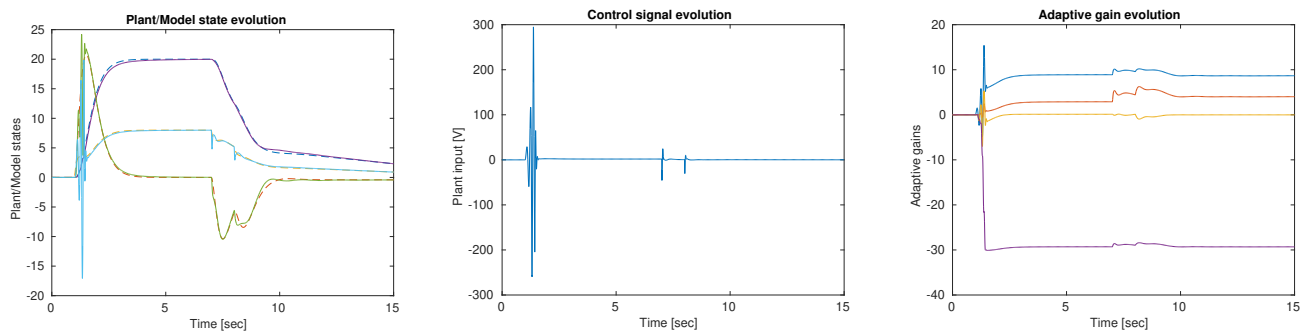

Figure 4: Response of adaptive control system without rate-limits: left, plant/model state evolution; centre, plant input $v$; right adaptive gains $\hat{K}_{x}, \hat{K}_{r}$

\section{CONCLUSION}

This paper has proposed a simple anti-windup scheme for MRAC with rate-limits. The scheme is inspired by the positive- $\mu$ scheme introduced in [14], but is developed in an anti-windup framework [21]. Unlike for standard anti-windup problems, where the difference between magnitude-limited and rate-limited anti-windup schemes are 

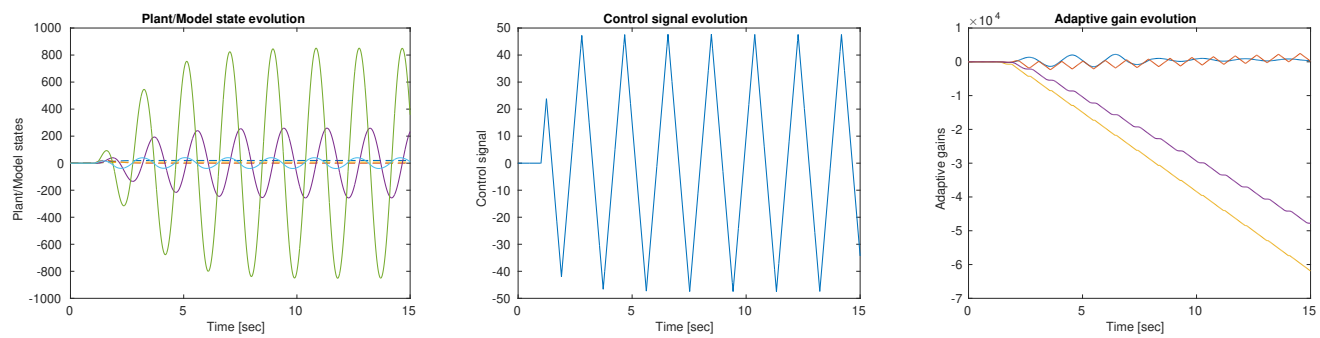

Figure 5: Response of adaptive control system with input rate-saturation and no anti-windup: left, plant/model state evolution; centre, plant input $v$; right adaptive gains $\hat{K}_{x}, \hat{K}_{r}$
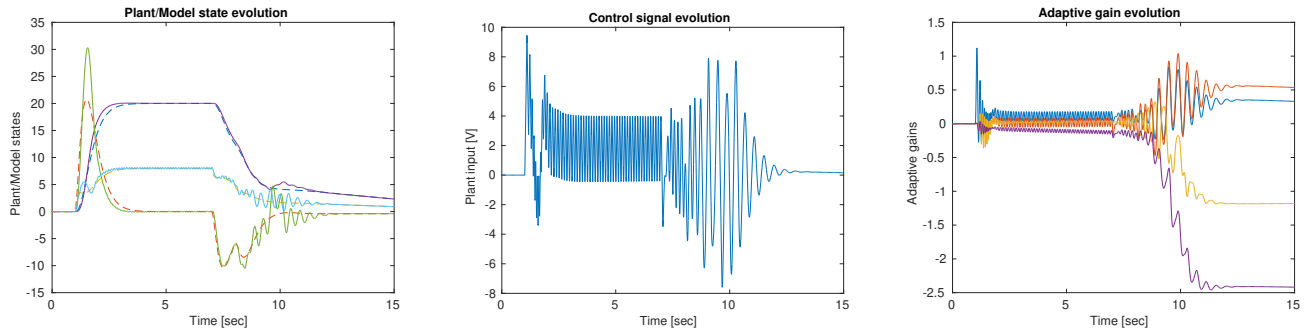

Figure 6: Response of adaptive control system with input saturation and anti-windup: left, plant/model state evolution; centre, plant input $v$; right adaptive gains $\hat{K}_{x}, \hat{K}_{r}$
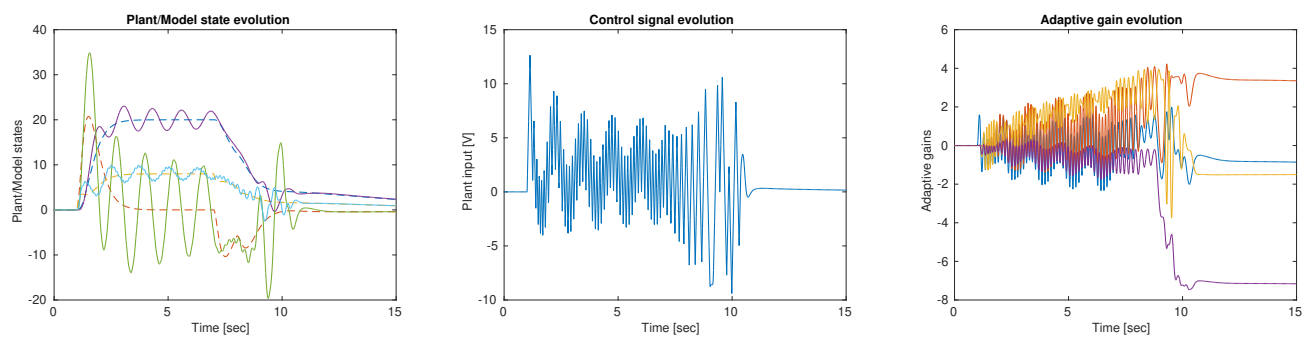

Figure 7: Response of adaptive control system with input saturation and anti-windup with $\hat{K}_{u} \equiv 0$ : left, plant/model state evolution; centre, plant input $v$; right adaptive gains $\hat{K}_{x}, \hat{K}_{r}$

relatively modest, in the case of MRAC, the differences are somewhat more profound and the hedging term, $B(\hat{x}-u)$, used in the scheme appears crucial, although this prevents the system from converging to exactly the same response as the ideal model. Numerical simulations have demonstrated the effectiveness of the scheme.

\section{ACKNOWLEDGEMENTS}

This research was supported by the UK Engineering and Physical Sciences Research Council, grant number EP/N00924X/1. 
[1] A.N. Annaswamy and S.P. Karason. Discrete-time adaptive control in the presence of input constraints. Automatica, 31(10):1421-1431, 1995.

[2] J.-M. Biannic and S. Tarbouriech. Optimisation and implementation of dynamic anti-windup compensators with multiple saturations in flight control systems. Control Eng Pract, 17(6):703-717, 2009.

[3] S. Galeani, S. Tarbouriech, M.C. Turner, and L. Zaccarian. A tutorial on modern anti-windup design. Eur J Control, 15(3-4):418-440, 2009.

[4] A.H. Glattfelder and W. Schaufelberger. Control systems with input and output constraints. Springer, London, 2003.

[5] B.C. Gruenwald, D. Wagner, T. Yucelen, and J.A. Muse. Computing actuator bandwidths for model reference adaptive control. Int. J. Control, 89(12):24342452, 2016.

[6] P. A. Ioannou and Jing S. Robust adaptive control. Courier Corporation, 2012.

[7] J.M. Gomes da Silva Jr., S. Tarbouriech, and G. Garcia. Local stabilization of linear systems under amplitude and rate saturating actuators. IEEE Transactions on Automatic Control, 48(5):842-847, 2003.

[8] E.N. Johnson. Limited Authority Adaptive Flight Control. PhD thesis, Georgia Institute of Technology, Georgia, November 2000.

[9] N. Kahveci, P.A. Ioannou, and M.M. Mirmirani. Adaptive LQ control with antiwindup augmentation to optimize UAV performance in autonomous soaring applications. IEEE Transactions on Control Systems Technology, 16(4):691-707, 2008.

[10] S.P. Karason and A.M. Annaswamy. Adaptive control in the presence of input constraints. IEEE Transactions on Automatic Control, 39:2325-2330, 1994.

[11] H. K. Khalil. Nonlinear Systems. MacMillan, 1992.

[12] H.K. Khalil. Nonlinear Systems. Prentice Hall, New Jersey, 1996.

[13] S.G. Khan, G. Herrmann, T. Pipe, C. Melhuish, and A. Spiers. Safe adaptive compliance control of a humanoid robotic arm with anti-windup compensation and posture control. International Journal of Social Robotics, 2(3):305-319, 2010.

[14] E. Lavretsky and N. Hovakimyan. Stable adaptation in the presence of input constraints. Systems and Control Letters, 56:722-729, 2007.

[15] N. A. Ofodile, M. C. Turner, and J. Sofrony. An alternative approach to antiwindup design for double integrator systems. In American Control Conference (ACC), 2016.

[16] A. Ortseifen and J. Adamy. A new design method for mismatch-based antiwindup compensators: Achieving local performance and global stability in the SISO case. In American Control Conference, 2011. 
[17] J. Sofrony, M.C. Turner, and I. Postlethwaite. Anti-windup using Riccati equations for systems with rate-limits. Int J Control, 83(2):233-245, 2010.

[18] S. Tarbouriech, G. Garcia, J. M. Gomes da Silva Jr., and I. Queinnec. Stability and Stabilization of Linear Systems with Saturating Actuators. Springer, 2011.

[19] S. Tarbouriech and M.C. Turner. Anti-windup design: an overview of some recent advances and some open problems. IET Control Theory A, 3(1):1-19, 2009.

[20] J.-F. Trégouët, D. Arzelier, D. Peaucelle, C. Pittet, and L. Zaccarian. Reaction wheels desaturation using magnetorquers and static input allocation. Control Systems Technology, IEEE Transactions on, 23(2):525-539, 2015.

[21] M.C. Turner. Positive $\mu$ modification as an anti-windup mechanism. Systems \& Control Letters, 102:15-21, 2017.

[22] F. Tyan and D. S. Bernstein. Global stabilization of systems containing a double integrator using a saturated linear controller. Int J Robust Nonlin, 9(15):11431156, 1999.

[23] J. Valasek, M. R. Akella, A. Siddarth, and E. Rollins. Adaptive dynamic inversion control of linear plants with control position constraints. Control Systems Technology, IEEE Transactions on, 20(4):918-933, 2012.

[24] K.A. Wise and E. Lavretsky. Adaptive control of flight: theory, applications, and open problems. In American Control Conference, Minneapolis, MN, 2006.

[25] L. Zaccarian and A.R. Teel. Modern Anti-windup Synthesis: Control Augmentation for Actuator Saturation. Princeton University Press, New Jersey, 2011.

\section{Appendix A. Proofs of preliminary results}

\section{Appendix A.1. Proof of Lemma 4}

First note that $\mathrm{Dz}_{\bar{u}}[\pi(t) u]$ and $\mathrm{Dz}_{\bar{u}}[\delta u]$ share the same sign for all $u(t)$ and any $\pi($. stipulated above. Next assume that $|\pi(t) u|<\bar{u}$, then $\mathrm{Dz}_{\bar{u}}[\pi(t) u]=0$ and hence the inequality in the lemma certainly holds. Finally, assume that $|\pi(t) u| \geq \bar{u}$. In this case we have

$$
\begin{aligned}
\left\|\mathrm{Dz}_{\bar{u}}[\pi(t) u]\right\| & =|\pi(t) u|-\bar{u} \\
& \leq \delta|u|-\bar{u} \\
& =\left\|\mathrm{Dz}_{\bar{u}}[\delta u]\right\|
\end{aligned}
$$

which completes the proof. 


\section{Appendix A.2. Proof of Lemma 5}

$V(x)=x^{\prime} P x$ implies that there exists postive constants $c_{1}$ and $c_{2}$ such that $c_{1}\|x\|^{2} \leq$ $V(x) \leq c_{2}\|x\|^{2}$. The inequality can also be bounded as

$$
\begin{aligned}
\dot{V}(x) & \leq-c_{3}\|x\|_{2}+\alpha_{2}\|x\| \sum_{i=1}^{m}\left\|u_{i}\right\| \\
& \leq-c_{4} V(x)+c_{5} \sqrt{V(x)} \sum_{i=1}^{m}\left\|u_{i}\right\|
\end{aligned}
$$

where $c_{3}, c_{4}$ and $c_{5}$ are appropriate positive constants. Now, similar to [12], Section 5.4, let $W(x)=\sqrt{V(x)}$ and note that $\dot{W}(x)=\dot{V}(x) / 2 \sqrt{V(x)}$. This then implies

$$
\dot{W}(x) \leq-\frac{c_{4}}{2} W(x)+\frac{c_{5}}{2} \sum_{i=1}^{m}\left\|u_{i}\right\|
$$

Application of the Comparison Lemma (Lemma 2.5, [12]) then implies that

$$
\|W(x)\| \leq e^{-\frac{c_{4}}{2} t}\|W(0)\|+\frac{c_{5}}{2} \int_{0}^{t} \sum_{i=1}^{m} e^{-\frac{c_{4}}{2}(t-\tau)}\left\|u_{i}(\tau)\right\| d \tau
$$

As $W(x)=\sqrt{x^{\prime} P x}$, this then implies there exist positive constants $c_{6}, c_{7}$ and $c_{8}$ such that

$$
\|x(t)\| \leq c_{7} e^{-c_{6} t}\|x(0)\|+c_{8} \int_{0}^{t} \sum_{i=1}^{m} e^{-c_{6}(t-\tau)}\left\|u_{i}(\tau)\right\| d \tau
$$

Then, following arguments similar to those found in [12] Section 6.1 and using Holder's Inequality, it follows that $x(t)$ converges to zero if $u_{i} \in \mathcal{L}_{2}$ for all $i \in\{1, \ldots, m\}$.

\section{Appendix B. Proofs of main results}

\section{Appendix B.1. Proof of Prop 1}

First consider the dynamics of the error between the plant state $x$ and the reference model state $x_{m}$

$$
\begin{aligned}
\dot{e}= & A x+B u+B\left(x_{a}-u\right)-A_{m} x_{m}-B_{m} r-B\left(\hat{x}_{a}-u\right) \\
& +\mu B \mathrm{Dz}_{\bar{u}}\left[\eta\left(u-\hat{x}_{a}\right)\right]+B \hat{K}_{u}^{\prime} \mathrm{Dz}_{\bar{u}}\left[\eta\left(u-\hat{x}_{a}\right)\right]
\end{aligned}
$$

Substituting for $u$ and cancelling terms in $\mu$ yields

$$
\begin{aligned}
\dot{e}= & A x+B\left\{K_{x}^{*} x+K_{r}^{*} r+\Delta K_{x}^{\prime} x+\Delta K_{r}^{\prime} r\right\}+B\left(x_{a}-u\right) \\
& -B\left(\hat{x}_{a}-u\right)-A_{m} x_{m}-B_{m} r+B \hat{K}_{u}^{\prime} \mathrm{Dz}_{\bar{u}}\left[\eta\left(u-\hat{x}_{a}\right)\right]
\end{aligned}
$$


Noting that $\left(A+B K_{x}^{*}\right)=A_{m}, B K_{r}^{*}=B_{m}$ and $e_{a}=x_{a}-\hat{x}_{a}$, the following expression for the error dynamics is obtained:

$$
\dot{e}=A_{m} e+B\left(\Delta K_{x}^{\prime} x+\Delta K_{r}^{\prime} r\right)+B e_{a}+B \hat{K}_{u}^{\prime} \mathrm{Dz}_{\bar{u}}\left[\eta\left(u-\hat{x}_{a}\right)\right]
$$

Also, the actuator error dynamics can be written as

$$
\begin{aligned}
\dot{e}_{a} & =\dot{x}_{a}-\dot{\hat{x}}_{a} \\
& =-\lambda_{1} e_{a}+\operatorname{sat}_{\bar{u}}\left[\eta\left(u-x_{a}\right)\right]-\operatorname{sat}_{\bar{u}}\left[\eta\left(u-\hat{x}_{a}\right)\right]-B^{\prime} P e
\end{aligned}
$$

Now consider a Lyapunov candidate function

$$
V=e^{\prime} P e+\Delta K_{x}^{\prime} \Gamma_{x}^{-1} \Delta K_{x}+\Delta K_{r}^{\prime} \Gamma_{r}^{-1} \Delta K_{r}+\hat{K}_{u}^{\prime} \Gamma_{u}^{-1} \hat{K}_{u}+e_{a}^{2}
$$

The derivative of the Lyapunov function is then given by:

$$
\begin{aligned}
\dot{V}= & 2 e^{\prime} P\left\{A_{m} e+B\left(\Delta K_{x}^{\prime} x+\Delta K_{r}^{\prime} r\right)+B e_{a}\right. \\
& \left.+B \hat{K}_{u}^{\prime} \mathrm{Dz}_{\bar{u}}\left[\eta\left(u-\hat{x}_{a}\right)\right]\right\} \\
& +2 \Delta K_{x}^{\prime} \Gamma_{x}^{-1} \dot{\hat{K}}_{x}+2 \Delta K_{r}^{\prime} \Gamma_{r}^{-1} \dot{\hat{K}}_{r}+2 \hat{K}_{u} \Gamma_{u}^{-1} \dot{\hat{K}}_{u} \\
& -2 e_{a}\left\{\lambda_{1} e_{a}-\operatorname{sat}_{\bar{u}}\left[\eta\left(u-x_{a}\right)\right]+\operatorname{sat}_{\bar{u}}\left[\eta\left(u-\hat{x}_{a}\right)\right]+B^{\prime} P e\right\} \\
= & e^{\prime}\left(A_{m}^{\prime} P+P A_{m}\right) e+2 e^{\prime} P B\left(\Delta K_{x}^{\prime} x+\Delta K_{r}^{\prime} r\right) \\
& +2 e^{\prime} P B \hat{K}_{u}^{\prime} \mathrm{Dz}_{\bar{u}}\left[\eta\left(u-\hat{x}_{a}\right)\right] \\
& -2 \Delta K_{x}^{\prime} x\left(e^{\prime} P B\right)-2 \Delta K_{r}^{\prime} r\left(e^{\prime} P B\right) \\
& -2 \hat{K}_{u} \mathrm{Dz}_{\bar{u}}\left[\eta\left(u-\hat{x}_{a}\right)\right]\left(e^{\prime} P B\right) \\
& +2 e_{a}^{\prime}\left\{-\lambda_{1} e_{a}+\operatorname{sat}_{\bar{u}}\left[\eta\left(u-x_{a}\right)\right]-\operatorname{sat}_{\bar{u}}\left[\eta\left(u-\hat{x}_{a}\right)\right]\right\} \\
= & e^{\prime}\left(A_{m}^{\prime} P+P A_{m}\right) e+2 e^{\prime} P B\left(\Delta K_{x}^{\prime} x+\Delta K_{r}^{\prime} r\right) \\
& -2\left(\Delta K_{x}^{\prime} x+\Delta K_{r}^{\prime} r\right)\left(e^{\prime} P B\right) \\
& +2 e_{a}^{\prime}\left\{-\lambda_{1} e_{a}+\operatorname{sat}_{\bar{u}}\left[\eta\left(u-x_{a}\right)\right]-\operatorname{sat}_{\bar{u}}\left[\eta\left(u-\hat{x}_{a}\right)\right]\right\} \\
= & -e^{\prime} Q e-2 \lambda_{1} e_{a}^{2}+2 e_{a}^{\prime}\left\{\operatorname{sat}_{\bar{u}}\left[\eta\left(u-x_{a}\right)\right]-\operatorname{sat}_{\bar{u}}\left[\eta\left(u-\hat{x}_{a}\right)\right]\right\}
\end{aligned}
$$

Next, define $u_{1}=x_{a}-u$ and $u_{2}=\hat{x}_{a}-u$. Note that $e_{a}$ can be expressed as $e_{a}=u_{1}-u_{2}$, which means that

$$
\begin{aligned}
\dot{V} & =-e^{\prime} Q e-\lambda_{1} e_{a}^{2}+2 \frac{1}{\eta} \eta\left(u_{1}-u_{2}\right)^{\prime}\left\{\operatorname{sat}_{\bar{u}}\left[-\eta u_{1}\right]-\operatorname{sat}_{\bar{u}}\left[-\eta u_{2}\right]\right\} \\
& =-e^{\prime} Q e-\lambda_{1} e_{a}^{2}-2 \frac{1}{\eta} \eta\left(u_{2}-u_{1}\right)^{\prime}\left\{\operatorname{sat}_{\bar{u}}\left[\eta u_{2}\right]-\operatorname{sat}_{\bar{u}}\left[\eta u_{1}\right]\right\}
\end{aligned}
$$

Now because $\operatorname{sat}_{\bar{u}}($.$) is slope restricted within the interval [0,1]$, Fact 1 implies that

$$
\begin{aligned}
-\eta\left(u_{2}-u_{1}\right)^{\prime}\left\{\operatorname{sat}_{\bar{u}}\left[\eta u_{2}\right]-\operatorname{sat}_{\bar{u}}\left[\eta u_{1}\right]\right\} \leq \\
-\left\{\operatorname{sat}_{\bar{u}}\left[\eta u_{2}\right]-\operatorname{sat}_{\bar{u}}\left[\eta u_{1}\right]\right\}^{2}
\end{aligned}
$$


Therefore

$$
\begin{aligned}
\dot{V} & \leq-e^{\prime} Q e-2 \lambda_{1} e_{a}^{2}-2 \frac{1}{\eta}\left\{\operatorname{sat}_{\bar{u}}\left[\eta u_{2}\right]-\operatorname{sat}_{\bar{u}}\left[\eta u_{1}\right]\right\}^{2} \\
& \leq-e^{\prime} Q e-2 \lambda_{1} e_{a}^{2}
\end{aligned}
$$

So far, only negative semi-definiteness of $\dot{V}$ has been proved and hence (B.2) only implies that $e, e_{a}, \hat{K}_{x}, \hat{K}_{r}$ and $\hat{K}_{u}$ are bounded. In order to prove convergence of $e(t)$ and $e_{a}(t)$, we use Lemma 3. Inequality (B.2) implies that $e(t)$ and $e_{a}(t)$ are in $\mathcal{L}_{\infty}$. Also note that $V(t)$ is bounded from below and non-increasing, which implies $\lim _{t \rightarrow \infty} V(t)=V(\infty)<\infty$. Furthermore, inequality (B.2) implies that

$$
\|\tilde{e}\|^{2} \leq-\frac{1}{\lambda_{\min }(\tilde{Q})} \dot{V}(t)
$$

where $\tilde{e}=\left[\begin{array}{ll}e^{\prime} & e_{a}^{\prime}\end{array}\right]^{\prime}$ and $\tilde{Q}=\operatorname{diag}\left(Q, 2 \lambda_{1}\right)$. Using this,

$$
\int_{0}^{\infty}\|\tilde{e}\|^{2} d t \leq \int_{0}^{\infty}\left(\frac{\dot{V}(t)}{-\lambda_{\min }(\tilde{Q})}\right) d t=\frac{V(0)-V(\infty)}{\lambda_{\min }(\tilde{Q})}
$$

which shows that $e(t) \in \mathcal{L}_{2}$ and $e_{a}(t) \in \mathcal{L}_{2}$. Thus convergence follows if $\dot{e}$ and $\dot{e}_{a}$ are both themselves bounded. Unfortunately, boundedness of $e$ and $e_{a}$ does not imply boundedness of $x, x_{a}, \hat{x}_{a}$ or $u$, hence this condition must be further investigated. The expressions for $\dot{e}$ and $\dot{e}_{a}$ are

$$
\begin{aligned}
\dot{e} & =A_{m} e+B e_{a}+B\left(\Delta K_{x}^{\prime} x+\Delta K_{r}^{\prime} r\right)+B \hat{K}_{u} \mathrm{Dz}_{\bar{u}}\left[\eta\left(u-x_{a}\right)\right] \\
\dot{e}_{a} & =-\lambda_{1} e_{a}+\operatorname{sat}_{\bar{u}}\left[\eta\left(u-x_{a}\right)\right]-\operatorname{sat}_{\bar{u}}\left[\eta\left(u-\hat{x}_{a}\right)\right]+B^{\prime} P e
\end{aligned}
$$

Because of boundedness of $e, e_{a}, \hat{K}_{x}, \hat{K}_{r}$ and $\hat{K}_{u}$ and because $r$ is bounded by assumption, it is clear that $\dot{e} \in \mathcal{L}_{\infty}$ if $u, x_{a}, \hat{x}_{a}$ and $x$ are themselves bounded. Boundedness of these signals is proved next.

- Consider the dynamics of the actuator

$$
\dot{x}_{a}=-\lambda_{1} x_{a}+\operatorname{sat}_{\bar{u}}\left[\eta\left(u-x_{a}\right)\right]
$$

Direct application of Lemma 2 implies that $x_{a}$ is bounded.

- Noting that $\hat{x}_{a}=e_{a}+x_{a}$, then $\hat{x}_{a}$ is bounded because $e_{a}$ and $x_{a}$ are bounded.

- The plant dynamics are given by

$$
\dot{x}=A x+B x_{a}
$$

Because $x_{a}$ is bounded, application of Lemma 1 implies that $x$ is also bounded. 
- The deadzone nonlinearity can be written as

$$
\operatorname{Dz}_{\bar{u}}(v)=\beta(v) v \quad \beta(.): \mathbb{R} \mapsto[0,1)
$$

Using this, the equation for the control signal becomes

$$
\begin{aligned}
u & =\hat{K}_{x}^{\prime} x+\hat{K}_{r}^{\prime} r-\mu \beta(.) \eta\left(u-\hat{x}_{a}\right) \\
& =\frac{1}{1+\mu \eta \beta(.)}\left(\hat{K}_{x}^{\prime} x+\hat{K}_{r}^{\prime} r+\mu \beta(.) \eta \hat{x}_{a}\right)
\end{aligned}
$$

where the first term on the right exists because $\mu$ is assumed to be positive, $\eta$ is also a positive constants and $\beta(.) \in[0,1)$. It is clear that $u$ is bounded because all terms on the right hand side are bounded.

As a result, it is possible to conclude that $\dot{e} \in \mathcal{L}_{\infty}$ and $\dot{e}_{a} \in \mathcal{L}_{\infty}$. Together with $e, e_{a} \in \mathcal{L}_{\infty} \cup \mathcal{L}_{2}$ this implies, by Lemma 3, that both signals converge to zero.

\section{Appendix B.2. Proof of Proposition 2}

The proof is quite long, simply due to the algebra, so is broken down into shorter steps. In addtion, to simplify the algebra further, the case where $\mu \equiv 0$ is proved. When $\mu \neq 0$, the essence of the proof remains the same but the algebra becomes more lengthy.

Step 1: $e_{r}$ dynamics. The $e_{r}$ dynamics can be written

$$
\begin{aligned}
\dot{e}_{r} & =-\lambda_{1} e_{r}+\operatorname{sat}_{\bar{u}}\left[\eta\left(u-\hat{x}_{a}\right)\right]+\eta\left(\hat{x}_{a r}-u\right)+B^{\prime} P e \\
& =-\left(\lambda_{1}+\eta\right) e_{r}-\operatorname{Dz}_{\bar{u}}\left[\eta\left(u-\hat{x}_{a}\right)\right]+B^{\prime} P e
\end{aligned}
$$

Using the partition (28), $u(t)=u^{*}(t)+\Delta u(t)$ so

$$
\begin{aligned}
\dot{e}_{r}=- & \left(\lambda_{1}+\eta\right) e_{r}-\mathrm{Dz}_{\bar{u}}\left[\eta\left(u^{*}+\Delta u-\hat{x}_{a}\right)\right]+B^{\prime} P e \\
=- & \left(\lambda_{1}+\eta\right) e_{r}-\left\{\mathrm{Dz}_{\bar{u}}\left[\eta\left(u^{*}+\Delta u-\hat{x}_{a}\right)\right]-\mathrm{Dz}_{\bar{u}}\left[\eta\left(u^{*}-\hat{x}_{a}\right)\right]\right\} \\
& \quad-\mathrm{Dz}_{\bar{u}}\left[\eta\left(u^{*}-\hat{x}_{a}\right)\right]+B^{\prime} P e
\end{aligned}
$$

Step 2: Lyapunov function $e_{r}$. With

$$
V\left(e_{r}\right)=\frac{1}{2} e_{r}^{2}
$$

it follows that

$$
\begin{aligned}
\dot{V}\left(e_{r}\right)= & -\left(\lambda_{1}+\eta\right)\left\|e_{r}\right\|^{2}-e_{r}^{\prime}\left\{\operatorname{Dz}_{\bar{u}}\left[\eta\left(u^{*}+\Delta u-\hat{x}_{a}\right)\right]-\operatorname{Dz}_{\bar{u}}\left[\eta\left(u^{*}-\hat{x}_{a}\right)\right]\right\} \\
& -e_{r}^{\prime} \operatorname{Dz}\left(\eta\left(u^{*}-\hat{x}_{a}\right)\right)+e_{r}^{\prime} B^{\prime} P e \\
\leq & -\left(\lambda_{1}+\eta\right)\left\|e_{r}\right\|^{2}+\left\|e_{r}\right\| \eta\|\Delta u\|+\left\|e_{r}\right\|\left(\left\|B^{\prime} P\right\|\|e\|+\left\|\mathrm{Dz}_{\bar{u}}\left[\eta\left(u^{*}-\hat{x}_{a}\right)\right]\right\|\right)
\end{aligned}
$$


where the inequality follows from the Lipschitz property of the deadzone. Also,

$$
\begin{aligned}
\mathrm{Dz}_{\bar{u}}\left[\eta\left(u^{*}-\hat{x}_{a}\right)\right]= & \mathrm{Dz}_{\bar{u}}\left[\eta\left(\left(u^{*}-\hat{x}_{a}+\hat{x}_{a r}-\hat{x}_{a r}\right)\right]\right. \\
=\mathrm{Dz}_{\bar{u}}\left[\eta\left(\left(u^{*}-e_{r}-\hat{x}_{a r}\right)\right]\right. & \\
& -\mathrm{Dz}_{\bar{u}}\left[\eta\left(u^{*}-\hat{x}_{a r}\right)\right]+\mathrm{Dz}_{\bar{u}}\left[\eta\left(u^{*}-\hat{x}_{a r}\right)\right]
\end{aligned}
$$

From this it follows (Lipschitz property and noting $\eta>0$ ) that

$$
\left\|\mathrm{Dz}_{\bar{u}}\left[\eta\left(u^{*}-\hat{x}_{a}\right)\right]\right\| \leq \eta\left\|e_{r}\right\|+\left\|\mathrm{Dz}_{\bar{u}}\left[\eta\left(u^{*}-\hat{x}_{a r}\right)\right]\right\|
$$

Now from Fact 2, we have that $\hat{x}_{a r}=K(t)\left(u^{*}(t)+\Delta u(t)\right)$ so,

$$
\begin{aligned}
\left\|\mathrm{Dz}_{\bar{u}}\left[\eta\left(u^{*}-\hat{x}_{a}\right)\right]\right\| & \leq \eta\left\|e_{r}\right\|+\left\|\mathrm{Dz}_{\bar{u}}\left[\eta\left(u^{*}(1-K(t))+K(t) \Delta u\right)\right]\right\| \\
& \leq \eta\left\|e_{r}\right\|+\eta\|K(t)\|\|\Delta u\|+\left\|\mathrm{Dz}_{\bar{u}}\left[\eta u^{*}(1-K(t))\right]\right\|
\end{aligned}
$$

Thus the derivative of the Lyapunov function is bounded

$$
\begin{aligned}
\dot{V}\left(e_{r}\right) \leq & -\left(\lambda_{1}+\eta\right)\left\|e_{r}\right\|^{2}+\left\|e_{r}\right\| \eta\|\Delta u\|+\left\|e_{r}\right\|\left\|B^{\prime} P\right\|\|e\| \\
& +\eta\left\|e_{r}\right\|^{2}+\eta\|K(t)\|\left\|e_{r}\right\|\|\Delta u\|+\left\|e_{r}\right\|\left\|\mathrm{Dz}_{\bar{u}}\left[\eta u^{*}(1-K(t))\right]\right\| \\
= & -\lambda_{1}\left\|e_{r}\right\|^{2}+\eta(1+\|K(t)\|)\left\|e_{r}\right\|\|\Delta u\|+\left\|e_{r}\right\|\left\|B^{\prime} P\right\|\|e\| \\
& \quad+\left\|e_{r}\right\|\left\|\mathrm{Dz}_{\bar{u}}\left[\eta u^{*}(1-K(t))\right]\right\| \\
\leq & -\lambda_{1}\left\|e_{r}\right\|^{2}+2 \eta\left\|e_{r}\right\| \Delta u\|+\| e_{r}\|\| B^{\prime} P\|\| e\|+\| e_{r}\|\| \mathrm{Dz}_{\bar{u}}\left[\eta u^{*}(1-K(t))\right] \|
\end{aligned}
$$

Using, Lemma 4 , it then follows, for all $u(t) \in \mathbb{R}$, that

$$
\left\|\mathrm{Dz}_{\bar{u}}\left[\eta u^{*}(1-K(t))\right]\right\| \leq\left\|\mathrm{Dz}_{\bar{u}}\left[2 \eta u^{*}\right]\right\| \quad K(t) \in[-1,1]
$$

we finally have

$$
\dot{V}\left(e_{r}\right) \leq-\lambda_{1}\left\|e_{r}\right\|^{2}+2 \eta\left\|e_{r}\right\|\|\Delta u\|+\left\|e_{r}\right\|\left\|B^{\prime} P\right\|\|e\|+\left\|e_{r}\right\|\left\|\mathrm{Dz}_{\bar{u}}\left[2 \eta u^{*}\right]\right\|
$$

Now, because $e \in \mathcal{L}_{2}$, Lemma 5 implies that $\lim _{t \rightarrow \infty} e_{r}(t)=0$ if

$$
\mathrm{Dz}_{\bar{u}}\left[2 \eta u^{*}\right], \Delta u \in \mathcal{L}_{2}
$$

which are the conditions given in the proposition.

Step 3. $e_{m}$ dynamics. Using equations (12) and (24), the dynamics of $e_{m}$ become

$$
\begin{aligned}
\dot{e}_{m}= & A_{m} e_{m}+B e_{r}-\hat{K}_{u} \mathrm{Dz}_{\bar{u}}\left[\eta\left(u *+\Delta u-\hat{x}_{a}\right)\right] \\
= & A_{m} e_{m}+B e_{r}-\hat{K}_{u}\left\{\mathrm{Dz}_{\bar{u}}\left[\eta\left(u *+\Delta u-\hat{x}_{a}\right)\right]-\mathrm{Dz}_{\bar{u}}\left[\eta\left(u^{*}-\hat{x}_{a}\right)\right]\right\} \\
& \quad-\mathrm{Dz}_{\bar{u}}\left[\eta\left(u^{*}-\hat{x}_{a}\right)\right]
\end{aligned}
$$

Step 4. Lyapunov function $V\left(e_{m}\right)$. Choosing $V\left(e_{m}\right)=e_{m}^{\prime} P e_{m}$ we have

$$
\begin{gathered}
\dot{V}\left(e_{m}\right)=2 e_{m}^{\prime} P A_{m} e_{m}+B e_{r}-\hat{K}_{u}\left\{\mathrm{Dz}\left(\eta\left(u *+\Delta u-\hat{x}_{a}\right)\right)-\operatorname{Dz}\left(\eta\left(u^{*}-\hat{x}_{a}\right)\right)\right\} \\
\quad-\mathrm{Dz}_{\bar{u}}\left[\eta\left(u^{*}-\hat{x}_{a}\right)\right] \\
\leq-e_{m}^{\prime} Q e_{m}+2 e_{m}^{\prime} P B e_{r}+2\left\|e_{m}\right\|\|\eta P\|\left\|\hat{K}_{u}\right\| \Delta u \| \\
\quad+2\left\|e_{m}\right\|\|P\|\left\|\hat{K}_{u}(t)\right\|\left\|\mathrm{Dz}_{\bar{u}}\left[\eta\left(u^{*}-\hat{x}_{a}\right)\right]\right\|
\end{gathered}
$$


From equation (B.16) in the earlier part of the proof we have

$$
\left\|\mathrm{Dz}_{\bar{u}}\left[\eta\left(u^{*}-\hat{x}_{a}\right)\right]\right\| \leq \eta\left\|e_{r}\right\|+\eta\|K(t)\|\|\Delta u\|+\left\|D z_{\bar{u}}\left[\eta(1-K(t)) u^{*}\right]\right\|
$$

So we have

$$
\begin{aligned}
\dot{V}\left(e_{m}\right) \leq & -e_{m} Q e_{m}+2\left\|e_{m}\right\|\|P\|\left(\left\|B_{m}\right\|+\left\|K_{u}(t)\right\| \eta\right)\left\|e_{r}\right\| \\
& +2\left\|e_{m}\right\|\|\eta P\|\left\|K_{u}(t)\right\|(1+\|K(t)\|)\|\Delta u\| \\
& +2\left\|e_{m}\right\|\|P\|\left\|K_{u}(t)\right\|\left\|D z_{\bar{u}}\left[\eta(1-K(t)) u^{*}\right]\right\| \\
\leq & -e_{m} Q e_{m}+2\left\|e_{m}\right\|\|P\|\left(\left\|B_{m}\right\|+\left\|K_{u}(t)\right\| \eta\right)\left\|e_{r}\right\| \\
& +4\left\|e_{m}\right\|\|\eta P\|\left\|K_{u}(t)\right\| \Delta u\|+2\| e_{m}\|\| P\|\| K_{u}(t)\|\| D z_{\bar{u}}\left[2 \eta u^{*}\right] \|
\end{aligned}
$$

Note that $e_{r} \in \mathcal{L}_{2}$, so $e_{m}$ converges to zero, by Lemma 5 if

$$
\mathrm{Dz}_{\bar{u}}\left[2 \eta u^{*}\right], \Delta u \in \mathcal{L}_{2}
$$

which is, again precisely the condition given in the proposition. 